Article

\title{
Fatty Acid, Flavonol, and Mineral Composition Variability among Seven Macrotyloma uniflorum (Lam.) Verdc. Accessions
}

\author{
John Bradley Morris ${ }^{1, *}$, Ming Li Wang ${ }^{1}$, Michael A. Grusak ${ }^{2}$ and Brandon Tonnis ${ }^{1}$ \\ 1 USDA, ARS, Plant Genetic Resources Conservation Unit, 1109 Experiment St., Griffin, \\ GA 30223-1797, USA; E-Mails: MingLi.Wang@ars.usda.gov (M.L.W.); \\ Brandon.Tonnis@ars.usda.gov (B.T.) \\ 2 USDA, ARS, Children's Nutrition Research Center, Department of Pediatrics, Baylor College of \\ Medicine, 1100 Bates St., Houston, TX 77030, USA; E-Mail: Mike.Grusak@ars.usda.gov \\ * Author to whom correspondence should be addressed; E-Mail: Brad.Morris@ars.usda.gov; \\ Tel.: +1-770-229-3253; Fax: +1-770-229-3323.
}

Received: 5 February 2013; in revised form: 25 February 2013 / Accepted: 26 February 2013 / Published: 14 March 2013

\begin{abstract}
Horse gram [Macrotyloma uniflorum (Lam.) Verdc.] seeds containing high concentrations of fatty acids, flavonols and minerals should provide government, public and private organizations with a nutritious and healthy food for use by malnourished and food deprived people worldwide. Seeds from seven horse gram accessions, geographically adapted to Griffin, GA, USA were analyzed for fatty acid, flavonol, and mineral concentrations using gas chromatography, reverse-phase high performance liquid chromatography, and inductively coupled plasma-optical emission spectroscopy, respectively. Significant year effects occurred for stearic, oleic, linoleic, arachidic, gadoleic, and lignoceric acids. Oleic, linoleic, and linolenic acid ranged from $8.9 \%-16.8 \%$, $40.3 \%-45.6 \%$, and $11.6 \%-14.3 \%$, respectively, as percent of total fatty acids measured (total oil ranged from $2.32 \%$ to $2.87 \%$ ). Seed concentrations of myricetin, quercetin, and kaempferol ranged from 0-36 $\mu \mathrm{g} / \mathrm{g}$ DW, 0-27 $\mu \mathrm{g} / \mathrm{g} \mathrm{DW}$, and 240-316 $\mu \mathrm{g} / \mathrm{g} \mathrm{DW}$, respectively and the only year effect was observed for kaempferol among the horse gram accessions. Year effects were found for $\mathrm{Fe}, \mathrm{K}, \mathrm{Mg}, \mathrm{Mn}, \mathrm{Ni}$, and $\mathrm{S}$. Mean concentrations of macrominerals $(\mathrm{Ca}, \mathrm{K}, \mathrm{Mg}, \mathrm{P}$, and $\mathrm{S}$ ) and microminerals $(\mathrm{Cu}, \mathrm{Fe}, \mathrm{Mn}, \mathrm{Ni}$, and $\mathrm{Zn})$ ranged from $1.3-14 \mathrm{mg} / \mathrm{g} \mathrm{DW}$, and 1.0-95.0 $\mu \mathrm{g} / \mathrm{g} \mathrm{DW}$, respectively. Several correlations were observed among several fatty acids, flavonols, and minerals. The mono-unsaturated fatty acid, oleic acid correlated significantly with linoleic acid $(r=-0.64)$, arachidic acid $(r=-0.61), \mathrm{Ca}(r=0.50)$ and $\mathrm{Zn}(r=0.51$, all at $P<0.01)$. The flavonol, myricetin
\end{abstract}


correlated significantly with quercetin $(r=0.92, P<0.0001)$, while quercetin correlated with $\mathrm{Ca}(r=0.82, P<0.0001)$ and kaempferol correlated with $\mathrm{Mg}(r=0.61, P<0.01)$. Several mineral correlations were found including Fe with $\mathrm{K}(r=0.66)$ and $\mathrm{Mg}(r=0.56$, both at $P<$ 0.01). These seven horse gram accessions can be used in breeding programs to facilitate the production of superior cultivars with favorable fatty acid profiles, flavonol content, and mineral compositions.

Keywords: Macrotyloma uniflorum; horse gram; fatty acid; flavonol; mineral composition; variability

\section{Introduction}

Fatty acids, flavonols, and minerals are very important phytochemical constituents in legume seeds, providing many human health benefits. The essential fatty acid, linoleic acid, when combined in an optimum balance with $\alpha$-linolenic acid may slow the onset of Parkinson's and Alzheimer's diseases [1]. These fatty acids are important for healthy cell membrane formation and functional development of the brain and nervous system [2]. The flavonol, myricetin has been found to be a potential skin [3] and bladder cancer preventer [4], used in pancreatic cancer therapeutics [5], and a potential severe acute respiratory syndrome coronavirus inhibitor [6]. Human clinical trials have shown that the flavonol, quercetin, reduces blood pressure in hypertensive patients [7], improves endothelial function for beneficial cardiovascular effects [8], increases endurance without exercise training [9], and decreased the intensity of knee osteoarthritis symptoms when combined with glucosamine hydrochloride and chondroitin sulfate [10]. Several macro-minerals including $\mathrm{Na}, \mathrm{K}, \mathrm{Ca}, \mathrm{Mg}, \mathrm{S}, \mathrm{P}$, and $\mathrm{Cl}$ as well as the micro-minerals, $\mathrm{Fe}, \mathrm{Zn}, \mathrm{Cu}, \mathrm{Mn}, \mathrm{I}, \mathrm{F}, \mathrm{Se}, \mathrm{Mo}, \mathrm{Co}$ (in $\mathrm{B}_{12}$ ) are essential for human life [11]. Micro-mineral malnutrition is a very serious problem in Africa with deficiencies causing anemia and IQ reduction [12].

Horse gram (Macrotyloma uniflorum (Lam.) Verdc. is a minor legume used as a pulse crop in India [13] and has been found to be good nutritional quality [14]. Horse gram seeds have recently been shown to prevent atherosclerosis in rats and may be a potential functional food for the prevention of hyperlipidaemic atherosclerosis [15]. An $\alpha$-amylase inhibitor from horse gram seeds has recently been shown to have antihyperglycemic potential [16]. Extracts from horse gram plants have shown potential for treating several human infections [17].

There is limited information regarding fatty acid, flavonol, mineral, and genetic variation among horse gram accessions in the USDA, ARS germplasm collection. Thirty-two horse gram accessions are available in the USDA collection. However, only seven horse gram accessions were chosen because of their adaptation to our geographic region for optimum seed production. Our objectives were to evaluate these field grown accessions for fatty acid, flavonol, and mineral variability.

2. Results and Discussion 


\subsection{Fatty Acids}

The seed fatty acid percentages including the fatty acids stearic, oleic, linoleic, arachidic, gadoleic, and lignoceric, were influenced by year and accession (Table 1). Total oil (per g DW) ranged from $2.32 \%$ to $2.87 \%$. Palmitic and behenic acid compositions varied by accession, but not by year. Significant variation for fatty acid composition occurred among these horse gram accessions (Table 2). Oleic acid composition from all seven accessions ranged from $8.9 \%$ to $16.8 \%$ (as percent of total fatty acids) and seeds from PI 639027 (Nepal) produced the highest amount (16.8\%), while PI 174824 from India produced the least (8.9\%). Joshi et al. [18] reported oleic acid content ranging from $14.6 \%$ to $25.1 \%$ in nine horse gram genotypes, which are a little higher than what we reported in samples from the U.S. horse gram collection. However, Krishna et al. [19] reported that horse gram contained 14.9\% oleic acid, which was slightly lower than what we found in PI 639027 (16.8\%). We found oleic acid content averaging $13.6 \%$ for all horse gram accessions tested and Kadam et al. [13] found similar oleic acid levels $(13 \%)$ in horse gram seeds. More of the human essential fatty acid, linoleic acid (ranging from $40.3 \%$ to $45.6 \%$ ) was produced from these seven horse gram accessions than all other fatty acids. Joshi et al. [18] reported linoleic acid content ranging from $20 \%$ to $34.2 \%$, while Krishna et al. [19] recorded $37.8 \%$ linoleic acid from horse gram genotypes. These were generally lower than what we found for all seven horse gram accessions tested from the USDA collection, which averaged 43.3\% linoleic acid, or for horse gram lines reported by Kadam et al. [13] which averaged 44.6\% linoleic acid. Seeds from PI 174824 produced significantly higher amounts of linoleic acid (45.6\%) and another essential human fatty acid, linolenic acid (14.3\%), than all other accessions. Joshi et al. [18] recorded only modest linolenic acid content among nine horse gram accessions ranging from $0.64 \%$ to $1.79 \%$. However, Krishna et al. [19] and Kadam et al. [13] reported similar linolenic acid content at 13\% and $13.7 \%$, respectively, which was comparable to what we found among seven horse gram accessions (averaging 12.4\%). Most of the additional fatty acid compositions found from these seven accessions were fairly low except for palmitic acid which ranged from $21.6 \%$ to $25.4 \%$. Krishna et al. [19] reported a similar palmitic acid content (19.6\%); however, Joshi et al. [18] reported palmitic acid content exceeding ours by 20 to 30 percentage points. 
Table 1. Mean squares from analysis of variance of fatty acids (\%) in horse gram seeds harvested from seven accessions (A) grown in two years (Y).

\begin{tabular}{|c|c|c|c|c|c|c|c|c|c|c|c|}
\hline Source & df & $14: 0$ & 16:0 & 18:0 & $18: 1$ & $18: 2$ & $18: 3$ & 20:0 & 20:1 & $22: 0$ & $24: 0$ \\
\hline Year $(Y)$ & 1 & $0.008 *$ & 0.827 & $0.257 * * *$ & $21.542 * * *$ & $21.023 * * *$ & 1.016 & $0.008 * *$ & $0.006^{* *}$ & 0.124 & $0.043 * *$ \\
\hline Block (B) & 1 & 0.001 & 0.615 & 0.012 & $0.860 *$ & 0.718 & 0.31 & 0.0006 & 0.002 & 0.112 & 0.005 \\
\hline Accession (A) & 6 & $0.007 *$ & $6.235^{* * *}$ & $0.057 * *$ & $25.979 * * *$ & $10.080 * * *$ & $3.338 *$ & $0.009 * * *$ & $0.005 * *$ & $0.328 * *$ & $0.154 * * *$ \\
\hline $\mathrm{A} \times \mathrm{Y}$ & 6 & 0.001 & 0.125 & 0.005 & $2.115 * * *$ & $1.338^{*}$ & 2.089 & 0.0003 & 0.0005 & 0.042 & 0.012 \\
\hline
\end{tabular}

*Significant at $P=0.05 ; * *$ Significant at $P=0.01 ; * *$ Significant at $P<0.0001$.

Table 2. Mean oil (\% of DW) and fatty acid (\% of total fatty acids) percentages among seven horse gram accessions grown in 2009 and 2010.

\begin{tabular}{cccccccccccc}
\hline Acc.(PI) & Oil\% $^{\dagger}$ & $\mathbf{1 4 : 0}$ & $\mathbf{1 6 : 0}$ & $\mathbf{1 8 : 0}$ & $\mathbf{1 8 : 1}$ & $\mathbf{1 8 : 2}$ & $\mathbf{1 8 : 3}$ & $\mathbf{2 0 : 0}$ & $\mathbf{2 0 : 1}$ & $\mathbf{2 2 : 0}$ & $\mathbf{2 4 : 0}$ \\
\hline 212636 & $2.40 \mathrm{~cd}$ & $0.56 \mathrm{a}$ & $25.4 \mathrm{a}$ & $2.00 \mathrm{a}$ & $12.6 \mathrm{~d}$ & $42.5 \mathrm{~d}$ & $11.6 \mathrm{~b}$ & $0.57 \mathrm{a}$ & $0.26 \mathrm{bcd}$ & $2.53 \mathrm{a}$ & $1.97 \mathrm{a}$ \\
165594 & $2.45 \mathrm{c}$ & $0.52 \mathrm{ab}$ & $24.9 \mathrm{ab}$ & $2.03 \mathrm{a}$ & $12.6 \mathrm{~d}$ & $43.0 \mathrm{~cd}$ & $12.8 \mathrm{ab}$ & $0.52 \mathrm{~b}$ & $0.23 \mathrm{de}$ & $1.92 \mathrm{bc}$ & $1.49 \mathrm{~cd}$ \\
174824 & $2.32 \mathrm{e}$ & $0.52 \mathrm{ab}$ & $24.1 \mathrm{bc}$ & $1.86 \mathrm{~b}$ & $8.9 \mathrm{e}$ & $45.6 \mathrm{a}$ & $14.3 \mathrm{a}$ & $0.52 \mathrm{~b}$ & $0.20 \mathrm{e}$ & $2.49 \mathrm{a}$ & $1.57 \mathrm{bc}$ \\
345729 & $2.40 \mathrm{~cd}$ & $0.51 \mathrm{ab}$ & $23.9 \mathrm{~cd}$ & $1.93 \mathrm{ab}$ & $14.0 \mathrm{c}$ & $43.5 \mathrm{c}$ & $11.7 \mathrm{~b}$ & $0.48 \mathrm{c}$ & $0.25 \mathrm{~cd}$ & $2.18 \mathrm{ab}$ & $1.47 \mathrm{~cd}$ \\
163321 & $2.61 \mathrm{~b}$ & $0.49 \mathrm{bc}$ & $23.2 \mathrm{~d}$ & $1.84 \mathrm{bc}$ & $14.4 \mathrm{c}$ & $43.8 \mathrm{bc}$ & $12.1 \mathrm{~b}$ & $0.46 \mathrm{~cd}$ & $0.28 \mathrm{abc}$ & $1.96 \mathrm{bc}$ & $1.61 \mathrm{~b}$ \\
639027 & $2.36 \mathrm{de}$ & $0.46 \mathrm{bc}$ & $24.0 \mathrm{~cd}$ & $1.73 \mathrm{c}$ & $16.8 \mathrm{a}$ & $40.3 \mathrm{e}$ & $12.5 \mathrm{~b}$ & $0.47 \mathrm{~cd}$ & $0.30 \mathrm{a}$ & $1.79 \mathrm{c}$ & $1.40 \mathrm{~d}$ \\
174827 & $2.87 \mathrm{a}$ & $0.44 \mathrm{c}$ & $21.6 \mathrm{e}$ & $1.75 \mathrm{c}$ & $15.8 \mathrm{~b}$ & $44.4 \mathrm{~b}$ & $12.1 \mathrm{~b}$ & $0.44 \mathrm{~d}$ & $0.29 \mathrm{ab}$ & $1.89 \mathrm{bc}$ & $1.38 \mathrm{~d}$ \\
\hline
\end{tabular}

Means followed by different letters are significantly different $(P<0.0001) ;{ }^{\dagger}$ Even though oil data was not included in the 2 year analysis, we did however, include oil \%'s on a dry weight basis from these seven horse gram seed samples previously stored at $-18^{\circ} \mathrm{C}$. Analyses were conducted using ANOVA with 2 replications (each replication consisted of a duplicated sample) per accession. 


\subsection{Flavonols}

There were significant accession effects for myricetin, quercetin, and kaempferol concentrations in horse gram seeds (Table 3). Kaempferol was the only flavonol affected by year. The flavonol, myricetin ranged in concentration from 0 to $36 \mu \mathrm{g} / \mathrm{g}$ on a dry weight basis in seeds among these horse gram accessions (Table 4). The accession, PI 174827, produced a significantly higher concentration of myricetin $(36 \mu \mathrm{g} / \mathrm{g})$ than most of the other accessions. Sreerama et al. [20] reported myricetin concentrations in horse gram cotyledons, embryonic axes, and seed coats averaging $2.4 \mu \mathrm{g} / \mathrm{g}, 32.9 \mu \mathrm{g} / \mathrm{g}$, and $35.5 \mu \mathrm{g} / \mathrm{g}$ DW, respectively. Quercetin ranged in concentration from 0 to $27.2 \mu \mathrm{g} / \mathrm{g}$ and PI 174827 produced a significantly higher quercetin concentration $(27.2 \mu \mathrm{g} / \mathrm{g})$ than several of the other horse gram accessions. Even though Sreerama et al. [20] reported a quercetin concentration of $9.7 \mu \mathrm{g} / \mathrm{g}$ DW in horse gram cotyledons, much higher concentrations of quercetin were found in the embryonic axes $(113.4 \mu \mathrm{g} / \mathrm{g} \mathrm{DW})$ and seed coat $(130 \mu \mathrm{g} / \mathrm{g} \mathrm{DW})$ of horse gram. We report an average of $17.5 \mu \mathrm{g} / \mathrm{g}$ DW for five horse gram accessions only because both PI 212636 and PI 639027 produced quercetin below quantifiable limits when evaluated using HPLC, therefore quercetin values could not be given to either of these accessions. This is slightly below the average of $22.5 \mu \mathrm{g} / \mathrm{g}$ reported for quercetin concentrations among several common bean seeds [21], but very similar to what we reported for PI 174827 (27.2 $\mu \mathrm{g} / \mathrm{g}$

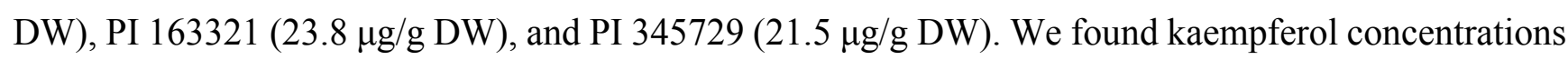
averaging $279 \mu \mathrm{g} / \mathrm{g} \mathrm{DW}$ in seven horse gram accessions, which was more than twice the concentration reported by Sreerama et al. [20] in horse gram seed coats and embryonic axes. However, they only found $9.7 \mu \mathrm{g} / \mathrm{g}$ DW of kaempferol in horse gram cotyledons. Kaempferol concentrations in common bean reported by Diaz-Batalla et al. [21] averaged $19.2 \mu \mathrm{g} / \mathrm{g}$ DW which was 15 times lower than what we found.

Table 3. Mean squares from analysis of variance of seed wt. (g) and flavonol concentration $(\mu \mathrm{g} / \mathrm{g} \mathrm{DW})$ of horse gram seeds harvested from seven accessions (A) and grown in two years (Y).

\begin{tabular}{cccccc}
\hline Source & Df & $\begin{array}{c}\text { Seed } \\
\mathbf{w t .}(\mathbf{g})\end{array}$ & Myricetin $(\boldsymbol{\mu g} / \mathbf{g})$ & $\begin{array}{c}\text { Quercetin } \\
(\boldsymbol{\mu g} / \mathbf{g})\end{array}$ & $\begin{array}{c}\text { Kaempferol } \\
(\boldsymbol{\mu g} / \mathbf{g})\end{array}$ \\
\hline Year(Y) & 1 & 0.00003 & 45.93 & 5.68 & $20040.15^{* * *}$ \\
Block(B) & 1 & 0.00002 & 3.98 & 0.94 & 153.53 \\
Accession(A) & 6 & 0.00002 & $524.98^{* *}$ & $171.66^{* *}$ & $3239.84 * *$ \\
AxY & 6 & 0.00003 & 26.82 & 11.84 & 549.3 \\
\hline \multicolumn{5}{c}{$*$ Significant at $P=0.01 ; * *$ Significant at $P<0.0001}$.
\end{tabular}


Table 4. Mean seed weight (g) and flavonol concentration ( $\mu \mathrm{g} / \mathrm{g} D W$ ) among seven horse gram accessions grown in 2009 and 2010.

\begin{tabular}{ccccc}
\hline Acc. $(\mathbf{P I})$ & $\begin{array}{c}\text { Seed } \\
\text { wt. }(\mathbf{g})\end{array}$ & $\begin{array}{c}\text { Myricetin } \\
(\boldsymbol{\mu g} / \mathbf{g})\end{array}$ & $\begin{array}{c}\text { Quercetin } \\
(\boldsymbol{\mu g} / \mathbf{g})\end{array}$ & $\begin{array}{c}\text { Kaempferol } \\
(\boldsymbol{\mu g} / \mathbf{g})\end{array}$ \\
\hline 174827 & $0.112 \mathrm{a}$ & $36.01 \mathrm{a}$ & $27.22 \mathrm{a}$ & $301.79 \mathrm{ab}$ \\
212636 & $0.111 \mathrm{a}$ & $0.00 \mathrm{c}$ & - & $315.53 \mathrm{a}$ \\
163321 & $0.110 \mathrm{a}$ & $26.55 \mathrm{ab}$ & $23.82 \mathrm{ab}$ & $283.34 \mathrm{bc}$ \\
165594 & $0.108 \mathrm{a}$ & $17.00 \mathrm{~b}$ & $14.88 \mathrm{c}$ & $240.36 \mathrm{e}$ \\
639027 & $0.106 \mathrm{a}$ & $0.00 \mathrm{c}$ & - & $247.65 \mathrm{de}$ \\
345729 & $0.106 \mathrm{a}$ & $25.65 \mathrm{ab}$ & $21.52 \mathrm{~b}$ & $293.31 \mathrm{abc}$ \\
174824 & $0.106 \mathrm{a}$ & $0.00 \mathrm{c}$ & $0.00 \mathrm{~d}$ & $271.73 \mathrm{~cd}$ \\
\hline
\end{tabular}

Means followed by different letters are significantly different $(P<0.0001)$.

\subsection{Minerals}

We reported year effects for several minerals including $\mathrm{Fe}, \mathrm{K}, \mathrm{Mg}, \mathrm{Mn}, \mathrm{Ni}$, and $\mathrm{S}$ (Table 5). Accession effects were observed for $\mathrm{Ca}$ and $\mathrm{S}$ only. Horse gram accessions differed significantly for the macro-minerals, $\mathrm{Ca}, \mathrm{P}$, and $\mathrm{S}$ (Table 6). We found nearly twice as high a concentration of $\mathrm{Ca}$ among seven horse gram accessions (averaging $2.4 \mathrm{mg} / \mathrm{g}$ ) than those reported by Kadam et al. [13]. Even though Ca was fairly low, PI 174827 accumulated a significantly higher concentration of Ca $(3.27 \mu \mathrm{g} / \mathrm{g})$ than PI 165594, PI 174824, PI 212636, and PI 639027. Horse gram accessions in our study averaged $13.6 \mathrm{mg} / \mathrm{g} \mathrm{DW}, 1.6 \mathrm{mg} / \mathrm{g} \mathrm{DW}, 4.1 \mathrm{mg} / \mathrm{g} \mathrm{DW}$, and $2.1 \mathrm{mg} / \mathrm{g} \mathrm{DW}$ of $\mathrm{K}, \mathrm{Mg}, \mathrm{P}$, and $\mathrm{S}$, respectively. Kadam et al. [13] reported similar levels of $\mathrm{Mg}$, but they did not report any other macro-mineral. There were no significant differences among horse gram accessions for the micro-minerals including $\mathrm{Cu}, \mathrm{Fe}$, and $\mathrm{Ni}$. We reported an average of $64 \mu \mathrm{g} / \mathrm{g}$ of $\mathrm{Mn}$ in horse gram seeds which was four times higher than what Kadam et al. [13] found. Only PI 212636 accumulated a significantly higher concentration of Mn $(95.25 \mu \mathrm{g} / \mathrm{g})$ than PI $174824(40.05 \mu \mathrm{g} / \mathrm{g})$. Zinc concentration averaged $37 \mu \mathrm{g} / \mathrm{g}$ among our horse gram accessions which was very similar to that found by Kadam et al. [13]. The accession, PI 639027 accumulated a significantly higher concentration of Zn $(42.14 \mu \mathrm{g} / \mathrm{g})$ than PI $212636(33.44 \mu \mathrm{g} / \mathrm{g})$ and PI $174824(33.21 \mu \mathrm{g} / \mathrm{g})$. Kadam et al. [13] found $55 \mu \mathrm{g} / \mathrm{g}$ and $119 \mu \mathrm{g} / \mathrm{g}$ of Cu and Fe, respectively in horse gram seeds which were much higher than our results. We found $\mathrm{Cu}$ and $\mathrm{Fe}$ concentrations among horse gram accessions averaging $11.5 \mu \mathrm{g} / \mathrm{g}$ and $71.2 \mu \mathrm{g} / \mathrm{g}$, respectively. Average Fe concentration exceeded all other micro-minerals, but was followed closely by Mn (averaging $64 \mu \mathrm{g} / \mathrm{g}$ ). Zinc concentrations averaged $36.7 \mu \mathrm{g} / \mathrm{g}$, while both PI $174824(33.21 \mu \mathrm{g} / \mathrm{g})$ and PI $212636(33.44 \mu \mathrm{g} / \mathrm{g})$ amassed significantly lower Zn concentrations than PI 639027 (42.14 $\mu \mathrm{g} / \mathrm{g})$. 
Table 5. Mean squares from analysis of variance of seed wt. (g) and mineral composition (macro-minerals are expressed as mg/g; micro-minerals are expressed as $\mu \mathrm{g} / \mathrm{g}$ dry weight $\mathrm{DW}$ ) of horse gram seeds harvested from seven accessions (A) and grown in two years (Y).

\begin{tabular}{|c|c|c|c|c|c|c|c|c|c|c|c|c|}
\hline Source & df & $\begin{array}{c}\text { Seed } \\
\text { wt. (g) }\end{array}$ & $\begin{array}{c}\mathrm{Ca} \\
(\mathrm{mg} / \mathrm{g})\end{array}$ & $\begin{array}{c}\mathbf{K} \\
(\mathrm{mg} / \mathrm{g})\end{array}$ & $\begin{array}{c}\mathrm{Mg} \\
(\mathrm{mg} / \mathrm{g})\end{array}$ & $\begin{array}{c}\mathbf{P} \\
(\mathrm{mg} / \mathrm{g})\end{array}$ & $\begin{array}{c}\mathrm{S} \\
(\mathrm{mg} / \mathrm{g})\end{array}$ & $\begin{array}{c}\mathrm{Cu} \\
(\mu \mathrm{g} / \mathrm{g})\end{array}$ & $\begin{array}{c}\mathbf{F e} \\
(\mu \mathrm{g} / \mathrm{g})\end{array}$ & $\begin{array}{c}\text { Mn } \\
(\mu \mathrm{g} / \mathrm{g})\end{array}$ & $\begin{array}{c}\mathrm{Ni} \\
(\mu \mathrm{g} / \mathrm{g})\end{array}$ & $\begin{array}{c}\mathrm{Zn} \\
(\mu \mathrm{g} / \mathrm{g})\end{array}$ \\
\hline Year (Y) & 1 & 0.000007 & $1.01^{*}$ & $14.78 * * *$ & $0.40 * * *$ & 0.13 & $0.14 * *$ & 6.04 & $1791.90 * *$ & $8216.10 * *$ & $3.88 * *$ & $72.00 *$ \\
\hline Block (B) & 1 & 0.0000003 & 0.32 & 0.34 & 0.003 & 0.08 & 0.01 & 2.62 & 99.57 & 2222.78 & 1.55 & 11.06 \\
\hline Accession (A) & 6 & 0.000005 & $2.11^{* * *}$ & 0.26 & 0.002 & 0.14 & $0.11 * *$ & 1.07 & 127.33 & 1659.97 & 0.44 & 32.61 \\
\hline $\mathrm{A} \times \mathrm{Y}$ & 6 & 0.000004 & 0.12 & 0.08 & 0.004 & 0.09 & 0.006 & 1.40 & 88.88 & 964.15 & 0.37 & 21.63 \\
\hline
\end{tabular}

*Significant at $P=0.05 ; * *$ Significant at $P=0.01 ; * *$ Significant at $P<0.0001$.

Table 6. Mean mineral composition (macro-minerals are expressed as $\mathrm{mg} / \mathrm{g}$; micro-minerals are expressed as $\mu \mathrm{g} / \mathrm{g}$ dry weight) among seven horse gram accessions grown in 2009 and 2010.

\begin{tabular}{cccccccccccc}
\hline Acc. (PI) & $\begin{array}{c}\text { Seed } \\
\mathbf{w t .}(\mathbf{g})\end{array}$ & $\begin{array}{c}\mathbf{C a} \\
(\mathbf{m g} / \mathbf{g})\end{array}$ & $\begin{array}{c}\mathbf{K} \\
(\mathbf{m g} / \mathbf{g})\end{array}$ & $\begin{array}{c}\mathbf{M g} \\
(\mathbf{m g} / \mathbf{g})\end{array}$ & $\begin{array}{c}\mathbf{P} \\
(\mathbf{m g} / \mathbf{g})\end{array}$ & $\begin{array}{c}\mathbf{S} \\
(\mathbf{m g} / \mathbf{g})\end{array}$ & $\begin{array}{c}\mathbf{C u} \\
(\boldsymbol{\mu g} / \mathbf{g})\end{array}$ & $\begin{array}{c}\mathbf{F e} \\
(\boldsymbol{\mu} \mathbf{g} / \mathbf{g})\end{array}$ & $\begin{array}{c}\mathbf{M n} \\
(\boldsymbol{\mu g} / \mathbf{g})\end{array}$ & $\begin{array}{c}\mathbf{N i} \\
(\boldsymbol{\mu} \mathbf{g} / \mathbf{g})\end{array}$ & $\begin{array}{c}\mathbf{Z n} \\
(\boldsymbol{\mu g} / \mathbf{g})\end{array}$ \\
\hline 212636 & $0.254 \mathrm{a}$ & $2.50 \mathrm{~b}$ & $13.26 \mathrm{a}$ & $1.61 \mathrm{a}$ & $3.97 \mathrm{bc}$ & $1.80 \mathrm{~d}$ & $11.75 \mathrm{a}$ & $63.47 \mathrm{a}$ & $95.25 \mathrm{a}$ & $1.83 \mathrm{a}$ & $33.44 \mathrm{~b}$ \\
163321 & $0.253 \mathrm{ab}$ & $3.00 \mathrm{ab}$ & $13.29 \mathrm{a}$ & $1.57 \mathrm{a}$ & $3.92 \mathrm{bc}$ & $2.00 \mathrm{c}$ & $11.94 \mathrm{a}$ & $75.28 \mathrm{a}$ & $63.06 \mathrm{ab}$ & $1.31 \mathrm{a}$ & $36.71 \mathrm{ab}$ \\
174827 & $0.253 \mathrm{ab}$ & $3.27 \mathrm{a}$ & $13.28 \mathrm{a}$ & $1.55 \mathrm{a}$ & $4.12 \mathrm{abc}$ & $2.12 \mathrm{bc}$ & $10.89 \mathrm{a}$ & $73.79 \mathrm{a}$ & $84.10 \mathrm{ab}$ & $1.81 \mathrm{a}$ & $36.81 \mathrm{ab}$ \\
345729 & $0.253 \mathrm{ab}$ & $2.91 \mathrm{ab}$ & $13.52 \mathrm{a}$ & $1.56 \mathrm{a}$ & $3.85 \mathrm{c}$ & $2.10 \mathrm{bc}$ & $12.08 \mathrm{a}$ & $79.37 \mathrm{a}$ & $46.63 \mathrm{ab}$ & $1.03 \mathrm{a}$ & $38.65 \mathrm{ab}$ \\
165594 & $0.253 \mathrm{ab}$ & $1.81 \mathrm{c}$ & $13.64 \mathrm{a}$ & $1.56 \mathrm{a}$ & $4.40 \mathrm{a}$ & $2.32 \mathrm{a}$ & $11.60 \mathrm{a}$ & $70.60 \mathrm{a}$ & $69.33 \mathrm{ab}$ & $1.89 \mathrm{a}$ & $36.00 \mathrm{ab}$ \\
174824 & $0.252 \mathrm{ab}$ & $1.32 \mathrm{c}$ & $13.84 \mathrm{a}$ & $1.59 \mathrm{a}$ & $4.11 \mathrm{abc}$ & $2.20 \mathrm{ab}$ & $10.93 \mathrm{a}$ & $66.39 \mathrm{a}$ & $40.05 \mathrm{~b}$ & $1.28 \mathrm{a}$ & $33.21 \mathrm{~b}$ \\
639027 & $0.251 \mathrm{~b}$ & $1.71 \mathrm{c}$ & $14.11 \mathrm{a}$ & $1.60 \mathrm{a}$ & $4.32 \mathrm{ab}$ & $2.23 \mathrm{ab}$ & $11.04 \mathrm{a}$ & $69.23 \mathrm{a}$ & $49.20 \mathrm{ab}$ & $1.53 \mathrm{a}$ & $42.14 \mathrm{a}$ \\
\hline
\end{tabular}

Means followed by different letters are significantly different $(P<0.0001)$. 


\subsection{Correlation Analysis}

Several fatty acids, flavonols, and minerals correlated significantly (Table 7). The saturated fatty acid, myristic acid showed highly significant positive correlations with palmitic acid $(r=0.81$, $P<0.0001)$; stearic acid $(r=0.77, P<0.0001)$; arachidic acid $(r=0.66, P<0.01)$ and $\mathrm{Cu}(r=0.51$, $P<0.01)$. Myristic acid also correlated negatively with oleic acid $(r=-0.53, P<0.01)$ and gadoleic acid $(r=-0.66, P<0.01)$. Palmitic acid showed a significantly positive correlation with stearic acid $(r=0.66$, $P<0.01)$ and arachidic acid $(r=0.73, P<0.001)$. However, palmitic acid also had a negative significant correlation with gadoleic acid $(r=-0.55, P<0.01)$; myricetin $(r=-0.69, P<0.01)$; quercetin $(r=-0.62, P<0.01)$; and $\mathrm{Ca}(r=-0.55, P<0.01)$. Therefore, as gadoleic acid, myricetin, and quercetin content of the seeds increased, palmtitic acid decreased. This is important because a breeding approach could focus on increasing both myricetin and quercetin content while decreasing palmitic acid. Stearic acid showed highly significant correlations with oleic acid $(r=-0.50)$; arachidic acid $(r=0.65)$; gadoleic acid $(r=-0.63) ; \mathrm{Cu}(r=0.59) ; \mathrm{K}(r=0.55)$; and $\mathrm{Mg}(r=0.51$, all at $P<0.01)$. Arachidic acid correlated significantly with behenic acid $(r=0.55)$; lignoceric acid $(r=0.62)$; myricetin $(r=-0.72)$, quercetin $(r=-0.79)$, and $\mathrm{Zn}(r=-0.54$, all at $P<0.01)$. As myricetin, quercetin, and $\mathrm{Zn}$ increased in horse gram seeds, arachidic acid decreased. Thus, a breeding approach could once again focus on increasing myricetin, quercetin, and $\mathrm{Zn}$ content while decreasing the saturated fatty acid, arachidic acid. Behenic acid correlated with lignoceric acid $(r=0.63, P<0.01)$ and lignoceric acid correlated with $\mathrm{S}(r=-0.76, P<0.0001)$. The mono-unsaturated fatty acid, oleic acid correlated, significantly with linoleic acid $(r=-0.64)$; arachidic acid $(r=-0.61)$; $\mathrm{Ca}(r=0.50) ; \mathrm{Zn}(r=0.51$, all at $P<0.01)$; gadoleic acid $(r=0.78)$ and quercetin $(r=0.83$, both at $P<0.0001)$. Another mono-unsaturated fatty acid, gadoleic acid, significantly correlated with quercetin $(r=0.64)$ and $\mathrm{Ca}(r=0.58$, both at $P<0.01)$. The poly-unsaturated fatty acid, linoleic acid, significantly correlated with both kaempferol and $\mathrm{Fe}(r=0.50$, $P<0.01)$, while linolenic acid had a significant negative correlation with both $\mathrm{Cu}(r=-0.66)$ and $\mathrm{Fe}$ ( $r=-0.50$, both at $P<0.01$ ). Both $\mathrm{Cu}$ and $\mathrm{Fe}$ in horse gram seeds can be increased in a breeding program, however linoleic acid would decrease. Myricetin correlated significantly with quercetin $(r=0.92)$, and $\mathrm{Ca}(r=0.82$, both at $P<0.0001)$, while quercetin correlated with $\mathrm{Ca}(r=0.82, P<$ $0.0001)$ and kaempferol correlated with $\mathrm{Mg}(r=0.61, P<0.01)$. Calcium negatively correlated with $\mathrm{K}$ $(r=-0.52, P<0.01)$ and $\mathrm{Cu}$ correlated with both $\mathrm{Fe}(r=0.71, P<0.0001)$ and $\mathrm{K}(r=0.56, P<0.01)$. Several additional mineral correlations were observed as well including Fe with $\mathrm{K}(r=0.66)$ and $\mathrm{Mg}$ ( $r=0.56$, both at $P<0.01)$; K with $\mathrm{Mg}(r=0.80, P<0.0001), \mathrm{Mn}(r=-0.53), \mathrm{S}(r=0.61, P<0.01)$; $\mathrm{Mg}$ with $\mathrm{Mn}(r=-0.53)$, Ni $(r=-0.59$, both at $P<0.01)$; Mn with Ni $(r=0.92, P<0.0001)$; and $\mathrm{P}$ with $\mathrm{S}$ $(r=0.63, P<0.01)$. These correlations provide evidence that these seven horse gram accessions consist of very important fatty acid profiles, flavonols, and minerals for use as parents in breeding programs and a potential functional food. 
Table 7. Correlation coefficients (r) resulting from Pearson correlation analysis of the mean compositions of ten fatty acids, three flavonols, and ten minerals in seven horse gram accessions. Statistical significance of correlation coefficient is indicated ${ }^{\dagger}$.

\begin{tabular}{|c|c|c|c|c|c|c|c|c|c|c|c|c|c|c|c|c|c|c|c|c|c|c|}
\hline & $16: 0$ & 18:0 & $18: 1$ & $18: 2$ & $18: 3$ & 20:0 & 20:1 & 22:0 & 24:0 & $\mathbf{m}$ & $\mathbf{q}$ & ka & $\mathrm{Ca}$ & $\mathrm{Cu}$ & $\mathrm{Fe}$ & $\mathbf{K}$ & Mg & Mn & $\mathrm{Ni}$ & $\mathbf{P}$ & $\mathbf{S}$ & $\mathrm{Zn}$ \\
\hline 14:0 & $0.81^{* * *}$ & $0.77^{* * * *}$ & $-0.53 * *$ & 0.16 & -0.22 & $0.66^{* *}$ & $-0.66^{* *}$ & $0.43^{*}$ & 0.34 & -0.41 & -0.46 & 0.24 & $-0.43^{*}$ & $0.51^{* *}$ & 0.24 & $0.42^{*}$ & $0.39 *$ & -0.31 & -0.31 & 0.01 & -0.007 & -0.26 \\
\hline $16: 0$ & & $0.66 * *$ & $-0.45^{*}$ & -0.18 & -0.09 & $0.73^{* * *}$ & $-0.55^{* *}$ & 0.38 & $0.45^{*}$ & $-0.69^{* *}$ & $-0.62^{* *}$ & -0.05 & $-0.55^{* *}$ & 0.36 & 0.02 & 0.27 & 0.23 & -0.12 & -0.06 & 0.2 & 0.002 & -0.08 \\
\hline 18:0 & & & $-0.5^{* *}$ & 0.33 & -0.3 & $0.65 * *$ & $-0.63^{* *}$ & 0.21 & 0.21 & -0.28 & -0.4 & $0.39 *$ & -0.29 & $0.59 * *$ & $0.46^{*}$ & $0.55^{* *}$ & $0.51 * *$ & -0.25 & -0.3 & 0.14 & 0.15 & -0.29 \\
\hline $18: 1$ & & & & $-0.64 * *$ & $-0.48^{*}$ & $-0.61 * *$ & $0.78^{* * *}$ & $-0.47^{*}$ & -0.23 & 0.42 & $0.83^{* * *}$ & -0.24 & $0.5^{* *}$ & -0.07 & -0.06 & -0.3 & -0.33 & 0.21 & 0.19 & -0.04 & -0.18 & $0.51^{* *}$ \\
\hline $18: 2$ & & & & & 0.09 & 0.03 & $-0.53^{*}$ & 0.17 & -0.13 & 0.23 & -0.13 & $0.50^{* *}$ & -0.01 & 0.27 & $0.50^{* *}$ & 0.35 & 0.35 & -0.24 & -0.29 & -0.01 & 0.23 & -0.30 \\
\hline $18: 3$ & & & & & & 0.11 & -0.15 & 0.007 & -0.13 & -0.16 & $-0.57 *$ & -0.23 & -0.34 & $-0.66^{* *}$ & $-0.50^{* *}$ & -0.13 & -0.09 & 0.01 & 0.12 & 0.04 & 0.26 & $-0.40^{*}$ \\
\hline 20:0 & & & & & & & $-0.46^{*}$ & $0.55^{* *}$ & $0.62^{* *}$ & $-0.72 * *$ & $-0.79 * *$ & 0.27 & $-0.45^{*}$ & 0.06 & -0.18 & 0.2 & 0.36 & -0.15 & -0.21 & -0.12 & -0.19 & $-0.54 * *$ \\
\hline $20: 1$ & & & & & & & & -0.36 & 0.02 & 0.36 & $0.64 * *$ & -0.1 & $0.58^{* *}$ & -0.34 & -0.27 & $-0.44^{*}$ & -0.33 & $0.41 *$ & 0.29 & -0.23 & $-0.4 *$ & 0.29 \\
\hline 22:0 & & & & & & & & & $0.63^{* *}$ & -0.32 & -0.48 & 0.09 & -0.23 & -0.007 & -0.25 & -0.17 & -0.17 & 0.009 & -0.01 & -0.29 & $-0.48^{*}$ & -0.12 \\
\hline 24:0 & & & & & & & & & & -0.41 & -0.02 & 0.16 & 0.04 & 0.03 & -0.37 & -0.34 & -0.13 & 0.38 & 0.23 & -0.36 & $-0.76^{* * *}$ & -0.23 \\
\hline $\mathrm{m}$ & & & & & & & & & & & $0.92 * * *$ & 0.1 & $0.82^{* * *}$ & -0.1 & 0.17 & $-0.49^{*}$ & -0.42 & 0.26 & 0.09 & -0.26 & -0.12 & 0.22 \\
\hline $\mathrm{q}$ & & & & & & & & & & & & 0.23 & $0.82^{* * *}$ & 0.08 & 0.06 & -0.32 & -0.27 & 0.32 & 0.15 & -0.2 & -0.47 & 0.38 \\
\hline ka & & & & & & & & & & & & & 0.24 & 0.24 & $0.44^{*}$ & 0.34 & $0.61^{* *}$ & -0.12 & -0.32 & -0.21 & -0.22 & -0.42 \\
\hline $\mathrm{Ca}$ & & & & & & & & & & & & & & -0.08 & 0.05 & $-0.52^{* *}$ & -0.25 & 0.37 & 0.1 & -0.38 & $-0.48^{*}$ & 0.1 \\
\hline $\mathrm{Cu}$ & & & & & & & & & & & & & & & $0.71^{* * *}$ & $0.56^{* *}$ & $0.41^{*}$ & -0.11 & -0.15 & 0.33 & 0.2 & 0.22 \\
\hline $\mathrm{Fe}$ & & & & & & & & & & & & & & & & $0.66^{* *}$ & $0.56^{* *}$ & -0.32 & -0.35 & 0.31 & $0.40^{*}$ & 0.20 \\
\hline $\mathrm{K}$ & & & & & & & & & & & & & & & & & $0.8^{* * *}$ & $-0.53^{* *}$ & $-0.45^{*}$ & $0.46^{*}$ & $0.61^{* *}$ & -0.1 \\
\hline $\mathrm{Mg}$ & & & & & & & & & & & & & & & & & & $-0.53^{* *}$ & $-0.59^{* *}$ & 0.24 & $0.38^{*}$ & -0.35 \\
\hline $\mathrm{Mn}$ & & & & & & & & & & & & & & & & & & & $0.91^{* * *}$ & 0.11 & $-0.42^{*}$ & 0.17 \\
\hline $\mathrm{Ni}$ & & & & & & & & & & & & & & & & & & & & 0.29 & -0.21 & 0.27 \\
\hline $\mathrm{P}$ & & & & & & & & & & & & & & & & & & & & & $0.63 * *$ & 0.29 \\
\hline $\mathrm{S}$ & & & & & & & & & & & & & & & & & & & & & & 0.13 \\
\hline
\end{tabular}

*Significant at $P=0.05 ; * *$ Significant at $P=0.01 ; * * *$ Significant at $P=0.0001 ;{ }^{\dagger}$ Oil $\%$ data was not included since it was not evaluated from field grown horse gram accessions. 


\section{Materials and Methods}

\subsection{Plant Materials}

Seven self-pollinating horse gram (Macrotyloma uniflorum (Lam.) Verdc. accessions from the USDA, ARS, Plant Genetic Resources Conservation Unit, Griffin, GA, USA were evaluated in this research. These seven accessions with plant introduction number, name, identifier, and origin are listed as follows: PI 163321, Kolt, 8484, (India); PI 165594, Ghat, 9404, (India); PI 174824, Ghat, 10015, (India); PI 174827, Ghat, 10180, (India); PI 212636, 13105, (India); PI 345729, (India); and PI 639027, GRIF 5517, I-905a, (Nepal). These seven accessions represent those horse gram samples adapted to the Griffin, GA environment for seed production.

Seed from each of these seven horse gram accessions were planted in $6.4 \mathrm{~cm} \times 7.0 \mathrm{~cm}$ jiffy pots (Hummert International, Earth City, MO, USA) containing Promix HP potting soil (Griffin Greenhouse, Ball Ground, GA, USA) each year (2009 and 2010) on April 1 and seedlings were grown in a greenhouse with no supplemental lighting at a temperature range of 21 to $26^{\circ} \mathrm{C}$. Seedlings were transplanted to the field on 5 May 2009 and 4 May 2010 in a randomized complete block design with 2 replications. The soil type for both the 2009 and 2010 evaluations was a clayey, kaolinitic, thermic typic kanhapludults soil series in Griffin, GA. A supplemental fertilizer consisting of a 10-10-10 NPK ratio was applied to the field soil prior to transplanting at a rate of $100 \mathrm{lbs} / \mathrm{acre}$. Twenty-five to 50 plants representing each accession per plot were transplanted in one $6 \mathrm{~m}$ row plot with $6 \mathrm{~m}$ between rows. Plots were irrigated using sprinklers as necessary. Mature pods were harvested from each horse gram accession 3 to 6 months after transplanting, dried at $21^{\circ} \mathrm{C}, 25 \% \mathrm{RH}$ for 1 week, and threshed.

\subsection{Fatty Acid Analysis}

Fatty acids were determined using an Agilent 7890A gas chromatograph (Agilent Technologies, Santa Clara, CA, USA) with a split/splitless inlet and flame ionization detector. Oil from ground horse gram seeds ( $250 \mathrm{mg}$ of seed meal) was extracted in $3 \mathrm{~mL}$ heptane and converted to fatty acid methyl esters (FAMEs) with $500 \mu \mathrm{L}$ of a $0.5 \mathrm{~N}$ sodium methoxide in methanol. An aliquot from the heptane layer was injected. Peak separations were performed on a DB-225 capillary column $(15 \mathrm{~m} \times 0.25 \mathrm{~mm}$ internal diameter with a $0.25 \mu \mathrm{m}$ film). One $\mu \mathrm{L}$ of sample was injected at a $15: 1$ ratio into the column using the following thermal gradient of: $195{ }^{\circ} \mathrm{C}$ for $1 \mathrm{~min}, 195$ to $200^{\circ} \mathrm{C}$ at $2.5^{\circ} \mathrm{C} / \mathrm{min}$ and 200 to $230{ }^{\circ} \mathrm{C}$ at $5{ }^{\circ} \mathrm{C} / \mathrm{min}$. The carrier gas was helium with an inlet pressure set to $12 \mathrm{psi}(\sim 1 \mathrm{~mL} / \mathrm{min}, \sim 39 \mathrm{~cm} / \mathrm{sec}$ at $\left.195^{\circ} \mathrm{C}\right)$. The peaks were identified by retention time comparison to a FAME standard mix RM-3 (Sigma-Aldrich, St. Louis, MO, USA) and the oven was equilibrated for 3.5 min between injections. Duplicate injections ensured adequate separation and quantification of all fatty acids in each sample. Two extractions and injections per replicated accession were used for data analysis.

\subsection{Flavonol Analysis}

Approximately $0.1 \mathrm{~g}$ of ground horse gram seed tissue was placed into tubes and $5 \mathrm{~mL}$ of extraction solvent consisting of $60 \%$ HPLC grade methanol with 1.2 M HCL was added to each sample, mixed, and incubated at $80{ }^{\circ} \mathrm{C}$ for $2 \mathrm{~h}$. The samples were then centrifuged, and part of the supernatant was filtered 
prior to injection. Separations were performed by reverse phase HPLC using a Zorbax Eclipse $3.0 \times$ $150 \mathrm{~mm}, 5 \mu \mathrm{m}, \mathrm{C} 18$ column (Agilent Technologies, Santa Clara, CA, USA) at $40{ }^{\circ} \mathrm{C}$ on an Agilent 1100 HPLC with a binary pump and autosampler. The sample injection volume was $5 \mu \mathrm{L}$, and analytes were monitored with a diode-array detector at $370 \mathrm{~nm}$ (flavonols). The absorption of $370 \mathrm{~nm}$ is the typical absorption wavelength for flavonols which are slightly different than isoflavonoids with a typical absorption near $285 \mathrm{~nm}$. This research is dealing with 3 flavonols including quercetin, kaempferol, and myricetin. Thus their absorption will be near the $370 \mathrm{~nm}$ range. Flavonol standards including myricetin (catalogue no. M6760), quercetin (catalogue no. Q4951) and kaempferol (catalogue no. 60010) (Sigma-Aldrich, St. Louis, MO, USA) were dissolved in a 5:3:2 mix of DMSO, methanol, water and diluted with $60 \%$ methanol to generate standard curves for peak identification and quantification. The mobile phase consisted of HPLC-grade acetonitrile (B) and $0.1 \%$ formic acid in filtered, sterile water (A). The flow rate was $0.8 \mathrm{~mL} / \mathrm{min}$ at the following gradient: $15 \% \mathrm{~B}$ at time zero to $35 \% \mathrm{~B}$ at $20 \mathrm{~min}$. The column was washed with $95 \% \mathrm{~B}$ for $5 \mathrm{~min}$ and equilibrated for $7 \mathrm{~min}$ between injections. The range of concentration for the linear calibration curve was 0.5 to $20 \mathrm{ng} / \mu \mathrm{L}$ for the flavonols. Duplicate extractions and injections in the mobile phase ensured adequate separation and quantification of all flavonols in each sample. Two extractions and injections per replicated accession were used for data analysis.

\subsection{Mineral Analysis}

Dried horse gram seed samples were ground to a fine powder using a stainless steel coffee grinder. A minimum of two sub-samples ( $\sim 0.25 \mathrm{~g} \mathrm{DW})$ from each ground sample were digested and processed for mineral analysis. Specifically, sub-samples were weighed and placed in $100 \mathrm{~mL}$ borosilicate glass tubes for pre-digestion overnight with $3 \mathrm{~mL}$ ultra-pure nitric acid. The following day, tubes were placed in a digestion block (Magnum Series; Martin Machine, Ivesdale, IL, USA) and maintained at $125{ }^{\circ} \mathrm{C}$ for a minimum of four $\mathrm{h}$ (with refluxing). Then, tubes were removed from the block, cooled for 5 min prior to adding $2 \mathrm{~mL}$ of hydrogen peroxide, and then they were returned to the block at $125{ }^{\circ} \mathrm{C}$ for $1 \mathrm{~h}$. This hydrogen peroxide procedure was repeated two more times. Finally, the digestion block temperature was raised to $200{ }^{\circ} \mathrm{C}$ and samples were maintained at this temperature until they were dry. Once cooled (after removal from the block), the digested samples were resuspended in $2 \%$ ultra-pure nitric acid overnight, then vortexed and transferred to plastic storage tubes until analysis for $\mathrm{Ca}, \mathrm{Cu}, \mathrm{Fe}, \mathrm{K}, \mathrm{Mg}, \mathrm{Mn}, \mathrm{Ni}, \mathrm{P}, \mathrm{S}$, and $\mathrm{Zn}$ concentrations. Mineral analysis was performed using ICP-OES (inductively coupled plasma-optical emission spectroscopy) (CIROS ICP Model FCE 12; Spectro, Kleve, Germany); the instrument was calibrated daily with certified standards. Tomato leaf standards (SRM 1573A; National Institute of Standards and Technology, Gaithersburg, MD, USA) were digested and analyzed along with the horse gram samples to ensure accuracy of the instrument calibration. Seed mineral concentrations were determined on a dry weight basis $(\mu \mathrm{g} / \mathrm{g}$ or $\mathrm{mg} / \mathrm{g}$ ), using an average value derived from the two sub-samples of each field replication.

\subsection{Statistical Analysis}

Data from the two year field experiments were combined to maximize the detection of accession differences over two years. The analysis of variance was performed using Proc GLM of SAS (SAS 9.2, 
SAS Institute, Inc., Cary, NC, USA) to determine significance of accession, year, and accession $\times$ year effects. Accession and year were treated as random effects. Mean separations were performed using Duncan's multiple range test $(P<0.0001)$. Correlations were analyzed using Proc Corr Pearson (SAS 9.2, SAS Institute, Inc., Cary, NC, USA).

\section{Conclusions}

These under-utilized horse gram accessions evaluated for various phytochemicals will provide breeders with valuable germplasm for the development of future cultivars with superior fatty acid, flavonol, and mineral concentrations for potential use as a functional food crop in the southeastern United States or in sub-tropical and tropical countries worldwide. Several of these accessions could also be grown by farmers for the production of a new summer pulse crop. Since more food will be required for sustaining a growing world population, horse gram can provide another healthy legume for consumption. Horse gram can also be used to help alleviate problems associated with malnutrition in Africa and Asia.

\section{References}

1. Youdim, K.A.; Martin, A.; Joseph, J.A. Essential fatty acids and the brain: Possible health implications. Int. J. Dev. Neurosci. 2000, 18, 383-399.

2. Brielmann, H.L.; Setzer, W.N.; Kaufman, P.B.; Kirakosyan, A.; Cseke, L.J. Natural Products from Plants, 2nd ed.; Cseke, L.J., Kirakosyan, A., Kaufman, P.B., Warber, S.L., Duke, J.A., Brielmann, H.L., Eds.; Taylor and Francis Group: Boca Raton, FL, USA, 2006; pp. 1-49.

3. Kang, N.J.; Jung, S.K.; Lee, K.W.; Lee, H.J. Myricetin is a potent chemopreventive phytochemical in skin carcinogenesis. Ann. N.Y. Acad. Sci. 2011, 1, 124-132.

4. Sun, F.; Zheng, X.Y.; Ye, J.; Wu, T.T.; Wang, J.I.; Chen, W. Potential anticancer activity of myricetin in human T24 bladder cancer cells both in vitro and in vivo. Nutr. Cancer 2012, 64, 599-606.

5. Phillips, P.A.; Sangwan, V.; Borja-Cacho, D.; Dudeja, V.; Vickers, S.M.; Saluja, A.K. Myricetin induces pancreatic cancer cell death via the induction of apoptosis and inhibition of the phosphatidylinositol 3-kinase (P13K) signaling pathway. Cancer Lett. 2011, 308, 181-188.

6. Yu, M.S.; Lee, J.; Lee, J.M.; Kim, Y.; Chin, Y.W.; Jee, J.G.; Keum, Y.S.; Jeong, Y.J. Identification of myricetin and scutellarein as novel chemical inhibitors of the SARS coronavirus helicase, nsP13. Bioorg. Med. Chem. Lett. 2012, 22, 4049-4054.

7. Edwards, R.L.; Lyon, T.; Litwin, S.E.; Rabovsky, A.; Symons, J.D.; Jalili, T. Quercetin reduces blood pressure in hypertensive subjects. J. Nutr. 2007, 137, 2405-2411.

8. Loke, W.M.; Hodgson, J.M.; Proudfoot, J.M.; McKinley, A.J.; Puddey, I.B.; Croft, K.D. Pure dietary flavonoids quercetin and (-)-epicatechin augment nitric oxide products and reduce endothelin-1 acutely in healthy men. Am. J. Clin. Nutr. 2008, 88, 1018-1025.

9. Davis, J.M.; Carlstedt, C.J.; Chen, S.; Carmichael, M.D.; Murphy, E.A. The dietary flavonoid quercetin increases VO(2max) and endurance capacity. Int. J. Sport Nutr. Exerc. Metab. 2010, 20, 56-62. 
10. Kanzaki, N.; Saito, K.; Maeda, A.; Kitagawa, Y.; Kiso, Y.; Watanabe, K.; Tomonaga, A.; Nagaoka, I.; Yamaguchi, H. Effect of a dietary supplement containing glucosamine hydrochloride, chondroitin sulfate and quercetin glycosides on symptomatic knee osteoarthiritis: A randomized, double-blind, placebo-controlled study. J. Sci. Food Agric. 2012, 92, 862-869.

11. Bouis, H.E.; Welch, R.M. Biofortification-A sustainable agricultural strategy for reducing micronutrient malnutrition in the global south. Crop Sci. 2010, 50, S20-S32.

12. Keatinge, J.D.H.; Waliyar, F.; Jamnadas, R.H.; Moustafa, A.; Andrade, M.; Drechsel, P.; Hughes, J.d'A.; Kadirvel, P.; Luther, K. Relearning old lessons for the future of food-By bread alone no longer: Diversifying diets with fruit and vegetables. Crop Sci. 2010, 50, S51-S62.

13. Kadam, S.S.; Salunkhe, D.K. Nutritional composition, processing, and utilization of horse gram and moth bean. Crit. Rev. Food Sci. Nutr. 1985, 22, 1-26.

14. Bravo, L.; Siddhuraju, P.; Saura-Calixto, F. Composition of underexploited Indian pulses. Comparison with common legumes. Food Chem. 1999, 64, 185-192.

15. Shobana, G.; Sivaelango, G.; Kumaravel, P. Evaluation of antihyperlipidaemic effect of macrotyloma uniflorum seed extract in experimental hyperlipidaemia. Res. J. Pharm. Technol. 2012, 5, 353-356.

16. Gupta, L.H.; Badole, S.L.; Bodhankar, S.L.; Sabharwal, S.G. Antidiabetic potential of $\alpha$-amylase inhibitor from the seeds of Macrotyloma uniflorum in streptozotocin-nicotinamide-induced diabetic mice. Pharm. Biol. 2011, 49, 182-189.

17. Kawsar, S.M.A.; Uddin, M.S.; Huq, E.; Nahar, N.; Ozeki, Y. Biological investigation of Macrotyloma uniflorum Linn. extracts against some pathogens. J. Biol. Sci. 2008, 8, 1051-1056.

18. Joshi, U.N.; Luthra, Y.P. Fatty acid profile of horse gram seeds. In Advances in Arid Legumes Research, Proceedings of the National Symposium on arid legumes, for food nutrition security and promotion of trade, Hisar, India, 15-16 May 2002.

19. Krishna, A.G.G.; Prabhakar, J.V.; Aitzetmuller, K. Tocopherol and fatty acid composition of some Indian pulses. J. Am. Oil Chem. Soc. 1997, 74, 1603-1606.

20. Sreerama, Y.N.; Sashikala, V.B.; Pratape, V.M. Variability in the distribution of phenolic compounds in milled fractions of chickpea and horse gram: Evaluation of their antioxidant properties. J. Agric. Food Chem. 2010, 58, 8322-8330.

21. Diaz-Batalla, L.; Widholm, J.M.; Fayey, G.C., Jr.; Castano-Tostado, E.; Paredes-Lopez, O. Chemical components with health implications in wild and cultivated Mexican common bean seeds (Phaseolus vulgaris L.). J. Agric. Food Chem. 2006, 54, 2045-2052.

(C) 2013 by the authors; licensee MDPI, Basel, Switzerland. This article is an open access article distributed under the terms and conditions of the Creative Commons Attribution license (http://creativecommons.org/licenses/by/3.0/). 